Revista de Derecho

de la Pontificia Universidad Católica de Valparaíso

53 (segundo semestre de 2019)

[pp. 125 - 145]

\title{
LA MASA ACTIVA DEL CONCURSO DE LA EMPRESA DE MENOR TAMAÑO. UNA APROXIMACIÓN BASADA EN LA REALIDAD EMPRESARIAL
}

[The insolvency state of the Micro, Small and Medium Enterprise. A Corporate View]

\author{
Lorena Carvajal Arenas* \\ Pontificia Universidad Católica de Valparaíso
}

Resumen

El presente artículo tiene por objeto principal ofrecer un esquema coherente para que el juez pueda definir cuál es el patrimonio de la Empresa de Menor Tamaño (EMT) que debe ser sometido a concurso. El tema es relevante por la posible intersección entre el patrimonio empresarial y aquel del empresario-persona natural. Esta situación, frecuente en la práctica, no está regulada en la legislación chilena, porque, aun cuando este tipo de empresas son vulnerables a caer en insolvencia, generalmente, las legislaciones no contemplan reglas especiales aplicables en dicha etapa crítica. Para lograr el objetivo indicado, se efectúa un estudio exegético y dogmático del soft law y de los estándares legales, jurisprudenciales y doctrinales de carácter nacional y de Derecho comparado (de los Estados Unidos, Italia y Canadá). Se concluye que el juez debe adoptar un criterio holístico, esto es, debe considerar aquellos bienes que pertenecen a la empresa -o sobre los cuales tiene algún derecho-y aquellos del patrimonio personal que forman parte del giro empresarial.

\section{Abstract}

This paper attempts to offer a coherent scheme to the judge in order to determine the insolvency estate of the Micro, small, and medium enterprises (MSMEs). This subject is relevant for the possible intersection between the company assets and those of the entrepreneur, as a natural person. This situation, frequent in practice, is not considered by Chilean law, because, even if MSEs are particularly vulnerable to becoming financially distressed, in general, national laws do not provide special rules for this critical stage in a MSMEs financial life. In order to attain the objective declared, an exegetical and dogmatic work takes place drawing on the soft law and legal, judicial and scholar standards from national and comparative sources (from the United States, Italy and Canada). It is pointed out, as a conclusion, that the judge must adopt a holistic approach, i.e., there must be considered corporate assets and the assets of the proprietor, which are used for running the business.

* PhD Universidad de Portsmouth. Profesora adjunta de Derecho Comercial. Pontificia Universidad Católica de Valparaíso. Dirección postal: Avenida Brasil 2950 Valparaíso. Dirección electrónica: lorena.carvajal@pucv.cl. Las traducciones desde el inglés e italiano al castellano fueron realizadas por la autora. 
Palabras clave

Derecho comercial - empresas de menor tamaño - insolvencia - patrimonio.
KeYwORDS

Commercial Law - micro, small, and medium enterprises - insolvency - assets.

Recibido el 10 de enero de 2019 y Aprobado el 13 de septiembre de 2019.

\section{INTRODUCCIÓN}

Conforme a la OCDE, "las pymes desempeñan un papel fundamental en el crecimiento económico, la creación de empleo, el desarrollo local y regional y la cohesión social. Prácticamente, todas las empresas de la zona de la OCDE son de pequeño o mediano tamaño, y generan en torno al 60\% del empleo total" . Debido a la importancia de la mipyme ${ }^{2}$ en la actividad económica y en la generación de empleo, se han desarrollado una serie de iniciativas legales destinadas a facilitar su constitución ${ }^{3}$ y también se han implementado políticas económicas generales, cuyo objeto es incentivar su productividad ${ }^{4}$. Sin embargo, la regulación aplicable durante la etapa de insolvencia de las Empresas de Menor Tamaño es insuficientes.

${ }^{1}$ OCDE, Financiamiento de pymes y emprendedores 2018: un marcador de la OCDE (2018); disponible [en línea]: https://www.oecd.org/cfe/smes/SPAHighlights-Financing-SMEs-and-Entrepreneurs-2018.pdf. En el ámbito doméstico, conforme a la Quinta Encuesta Longitudinal de Empresas de 2019, de un total de 339.022 empresas analizadas, las pymes fueron aquellas de mayor participación porcentual, con un $52,5 \%$. Le siguen las microempresas con un 44,4\%. Las grandes empresas sólo representaron un 3,1\%. Ministerio de EConomía, Fomento y Turismo, Unidad de Estudios. División de Política Comercial e Industrial (2019), disponible [en línea]: https://www.economia.gob.cl/2019/03/12/quinta-encuestalongitudinal-de-empresas-ele5.htm.

${ }^{2}$ El presente artículo utilizará indistintamente los términos mipyme, pyme y Empresa de Menor Tamaño (EMT). La equivalencia terminológica se inserta en el contexto de la legislación chilena. Conforme al Artículo Segundo de la Ley $\mathrm{N}^{\circ}$ 20.416, de 13 de enero de 2010: "Para los efectos de esta ley, se entenderá por empresas de menor tamaño las microempresas, pequeñas empresas y medianas empresas".

${ }^{3}$ Así, por ejemplo, en Chile: la Ley $\mathrm{N}^{\circ} 19.749$ que establece normas para facilitar la creación de microempresas familiares. Fecha de publicación 16 de mayo de 2002.

${ }^{4}$ Por ejemplo, el 17 de agosto de 2018 se da inicio a un proyecto de ley (Boletín $\mathrm{N}^{\circ}$ 12.025-03), el cual establece medidas para impulsar la productividad y el emprendimiento. Con todo, un estudio afirma que: "las políticas económicas generales habitualmente siguen manteniendo un sesgo que perjudica a las empresas de menor tamaño". Valenzuela, María Elena - Di Meglio, Roberto - Reinecke, Gerhard, La microempresa en Chile: la importancia del entorno regulatorio y desarrollo social, en Valenzuela, María Elena - Di Meglio, Roberto -Reinecke, Gerhard (eds.), De la Casa a la formalidad. Experiencias de la Ley de Microempresas Familiares en Chile (Santiago, Oficina Internacional del Trabajo, 2006), p. 41.

${ }^{5}$ Vid. Cirmizi, Elena - Klapper, Leora - Uttamchandani, Mahesh, The 
En Chile, la Ley No 20.416 del año 2010 (Estatuto Pyme) en su Artículo Undécimo fija un sistema de reorganización o cierre de micro y pequeñas empresas en crisis, es decir, excluye a las medianas empresas. Para ser encuadradas en esta normativa especial, tales empresas en crisis deben tributar en primera categoría de la Ley sobre Impuesto a la Renta ${ }^{6}$. El objetivo de esta Ley es ofrecer un sistema expedito de renegociación de la deuda y, consecuencialmente, favorecer la mantención de la empresa viable. Este procedimiento está centrado en la reorganización con efectos relativos entre las partes. Esta regulación es susceptible de crítica, al menos, desde dos perspectivas. Primero, por la ausencia de un procedimiento con efecto erga omnes que permita adoptar acuerdos vinculantes para todos los acreedores ${ }^{7}$. Segundo, por no contemplar un procedimiento liquidatorio, en circunstancias que sólo un número escaso de mipymes logra reestructurarse ${ }^{8}$. Ante la inviabilidad de la empresa, la opción que la Ley ofrece es el abandono de bienes por el deudor a los acreedores, lo cual devendrá en la extinción de la EMT. Como se aprecia, la legislación especial no ofrece una regulación adecuada. La alternativa disponible es la aplicación de los procedimientos contemplados en la Ley $\mathrm{N}^{\circ} 20.720$. Sin embargo, tampoco los procedimientos e instituciones contemplados en

challenges of bankruptcy reform, en The World Bank Development Research Group. Finance and Private Sector Development Team (2010), disponible [en línea]: https://papers.ssrn.com/sol3/papers.cfm?abstract_id=1694337. Naturalmente, una regulación adecuada en esta materia permitiría revertir las estadísticas que revelan un alto nivel de extinción de las empresas en los primeros años de vigencia: "Aproximadamente, el 60\% de la tasa de actividad emprendedora del grupo está compuesta por emprendimientos nacientes con menos de tres meses de operación, mientras que la tasa de discontinuidad empresarial es del $4 \%$ en la OECD y del $7 \%$ en Chile". Guerrero, Maribel - Serey, Tomás, Global Entrepreneurship Monitor. Reporte Nacional de Chile 2018 (2019), disponible: [en línea]: https://negocios.udd. cl/gemchile/files/2019/06/GEM-Reporte-Nacional-de-Chile-2018-1.pdf], p. 38

${ }^{6}$ Esta norma excluye a los profesionales. En la misma línea, el proyecto de ley (Boletín $N^{\circ}$ 12.025-03) en su Artículo Noveno. Cf. n. 62 de este trabajo.

${ }^{7}$ Un sistema eficiente de liquidación con efecto erga omnes es imperioso en el caso de la mipyme, dado que la escasez de activos para redestinar o vender y la dificultad para encontrar financiamiento en general y, especialmente, en esta fase, dificultan notablemente alcanzar un plan de reorganización exitoso. Vid. PugA, Juan Esteban, Derecho concursal. El acuerdo de reorganización (4a edición, Santiago, Editorial Jurídica de Chile, 2015), p. 14.

${ }^{8}$ Las estadísticas de la Superintendencia de Insolvencia y Reemprendimiento indican que, el año 2018, sólo 20 Asesorías Económicas de Insolvencia fueron finalizadas por la SIR. La información sobre este punto fue obtenida a través de requerimiento oficial al Consejo para la Transparencia el 25 marzo de 2019. Fecha de respuesta a la solicitud: 15 abril de 2019 . 
esa ley responden adecuadamente a las necesidades de la mipyme 9 . Entre los puntos críticos, cuya regulación no se aborda por estas leyes, es posible mencionar: el acceso temprano al procedimiento de reorganización ${ }^{10}$; la reducción de los requisitos de participación de los acreedores para hacer frente a su pasividad ante la apertura de una reorganización ${ }^{11}$; el acceso a procedimientos de liquidación rápidos y de bajo costo; el financiamiento de la administración del concurso; los aspectos relativos al llamado "nuevo comienzo" ${ }^{12} ; \mathrm{y}$, por último, el tratamiento de los bienes que constituyen la masa del concurso, lo cual constituye el objeto de este trabajo.

El tratamiento de los bienes que constituyen la masa activa de la liquidación de la mipyme reviste importancia por la confusión que, habitualmente, tiene lugar entre el patrimonio de la empresa y aquel del empresario. Ante la falta de regulación legal, esta investigación propone como teoría que, para determinar la masa concursal de la mipyme ${ }^{13}$, se requiere una interpretación del sistema jurídico que permita identificar los activos que

${ }^{9}$ En consideración a la especialidad de la mipyme, el artículo $1^{\circ}$ de la Legge Fallimentare de Italia excluye al pequeño emprendedor de la aplicación de los procedimientos concursales de quiebra e insolvencia, a través del establecimiento de ciertos límites. Por ejemplo, no se aplica la quiebra a quien "haya tenido en los tres ejercicios anteriores a la fecha de depósito de la instancia de quiebra un activo de monto anual no superior a 300.000 Euros".

${ }^{10}$ El elevado número de certificados de insolvencia emitidos (138) en relación con el escaso número de asesorías económicas de insolvencia válidamente iniciadas y terminadas (20) permite postular que se procura la utilización de este procedimiento para obtener los efectos ventajosos previstos en el artículo 18 de la Ley $\mathrm{N}^{\circ} 20.416$, esto es, la suspensión de: apremios de cualquier clase; actos que sean consecuencia directa del protesto de documentos mercantiles; los actos judiciales que impliquen embargos; los procedimientos o juicios de carácter tributario; y cualquier otra medida de carácter administrativo o judicial, incluso ante juzgados de policía local, entre otros. La información sobre este punto fue obtenida a través de requerimiento oficial por parte de la investigadora al Consejo para la Transparencia el 25 marzo de 2019. Fecha de respuesta a la solicitud: 15 abril de 2019.

${ }^{11} \mathrm{La}$ pasividad deviene de la carencia de bienes y apoyos necesarios para lograr el salvamento de la EMT.

${ }^{12}$ Sobre el nuevo comienzo o extinción de las obligaciones que constituyen el saldo no cubierto por la liquidación, vid.: Ruz, Gonzalo, Nuevo Derecho Concursal Chileno (Santiago, Thomson Reuters, 2017), II, p. 1254 ss.

${ }^{13}$ Fuera del concurso, la determinación del patrimonio de la EMT tiene relevancia a propósito del establecimiento de comercio, el cual, como es sabido no tiene regulación legal en Chile. En Italia, en cambio, el artículo 2055 del Codice Civile lo define como: "el conjunto de bienes organizados por el empresario para el ejercicio de la empresa”. La noción jurídica de "azienda”, está formada, conforme al artículo 2555 del Codice, no de bienes del empresario, sino de bienes "organizados por el empresario”. Esta es una noción muy parecida a la teoría que en este artículo se 
forman parte de la actividad empresarial, más allá de las fórmulas jurídicas que se utilicen para revestir tal actividad. De esta manera, el tema de estudio conduce hacia un aspecto esencial del Derecho concursal corporativo, esto es, qué se entiende por empresa en este contexto ${ }^{14}$. Una vez que se ha determinado que existe una empresa que opera con un determinado patrimonio, éste será parte de la masa concursal, aun cuando formalmente sea de propiedad del empresario como persona natural. El criterio a aplicar es holístico, basado en la realidad empresarial.

El artículo se divide en dos partes. En la primera, se estudia el soft law -el cual se alinea con la teoría propuesta en este trabajo- para determinar cómo sus prescripciones pueden aplicarse en el contexto nacional; especialmente, cuando la empresa ha asumido una personalidad jurídica. En la segunda parte, siempre en línea con la teoría propuesta y ante la falta de una definición legal sustancial, se ofrecen una serie de elementos que permiten determinar cuándo debe entenderse que opera una empresa en la realidad.

\section{CONSTITUCIÓN DE LA MASA ACTIVA DEL CONCURSO DE LA EMPRESA de MENOR TAMAÑo}

Las Empresas de Menor Tamaño se encuentran entre los principales usuarios de los sistemas concursales ${ }^{15}$. Esta situación tiene lugar debido a la necesidad de financiamiento que, casi siempre, asola a la pyme durante la etapa inicial ${ }^{16} ; y$, también, debido a la falta de capacidades estratégicas

presenta. Vid. Galgano, Francesco, Diritto Commerciale. L'emprenditore - Le società (8a edición, Bologna, Zanichelli, 2013), pp. 43 s.

${ }^{14}$ Es necesario determinar qué se entiende por empresa en esta sede, porque no existe una definición de la institución en el Derecho comercial codificado chileno. Vid. Carvajal, Lorena, La codificación del Derecho comercial chileno y sus ejes, en Revista Chilena de Derecho 44 (2017), 1, pp. 83-106. El argumento ha sido desarrollado por la Comisión de Estudio para la Nueva Codificación Comercial. Comisión DE ESTUdio NUEVA CODIFICACIÓn COMERCial, Sujetos del comercio y de los negocios, la empresa y sus elementos, en particular la propiedad intelectual (3a subcomisión, Universidad de Chile, Facultad de Derecho Departamento de Derecho Concursal, 2017), pp. 25-29, disponible [en línea]: http://codificacioncomercial.cl/wp-content/ uploads/2017/02/InformePreliminar-3aSubcomision.pdf.

${ }^{15}$ Así, International Bank for Reconstruction and Development - The WORLD BANK, Report on the Treatment of MSME Insolvency (2017), disponible [en línea]: http://documentos.bancomundial.org/curated/es/973331494264489956/ Report-on-the-treatment-of-MSME-insolvency. No hay traducción oficial para este documento del Banco Mundial. Tampoco se ofrece la traducción oficial del título del documento.

${ }^{16}$ Vid. Sarra, Janis, Micro, Small and Medium Enterprise (MSME) Insolvency 
de los emprendedores ${ }^{17}$. Ambos factores deben ser acometidos a través de políticas públicas adecuadas. Desde el punto de vista jurídico, se requiere contar con un procedimiento concursal accesible y fluido que permita identificar, proteger y reorganizar o liquidar los escasos activos de las pymes deudoras; $\mathrm{y}$, al mismo tiempo, tratar con la tensión financiera sin la pérdida de salvaguardias para los acreedores -entre ellos, los trabajadores ${ }^{18}{ }^{18}$. En ese contexto, el tratamiento de los bienes que constituyen la masa activa del concurso de la EMT es un punto crítico, porque la normativa aplicable -fundamentalmente, la Ley $\mathrm{N}^{\circ} 20.416$ y la Ley $\mathrm{N}^{\circ} 20.720$ - no regula adecuadamente el caso de confusión entre el patrimonio de la empresa y aquel del empresario. Esta situación es común en los casos en que la empresa no adopta un revestimiento jurídico que le otorga personalidad jurídica que la distingue del empresario-persona natural. Con todo, el caso también se presenta cuando existe una personalidad jurídica distinta del empresario $^{19}$. La confusión patrimonial encuentra una explicación en la infracapitalización. En Chile, este fenómeno es un factor muy importante en la búsqueda de financiamiento externo, porque no existe siquiera un

in Canada. Report for the Marketplace Policy Branch of Industry Canada, en Peter A. Allard School of Law Allard Research Commons, Faculty Publications (2016), pp. 29, disponible [en línea]: https://ssrn.com/abstract=2862039. El texto indica que, en una muestra aleatoria de mypimes insolventes en Canadá, la principal razón aducida para explicar la insolvencia es el uso de líneas de crédito personales.

17 "Existe una necesidad urgente de financiamiento, así como de educación y desarrollo de habilidades para individuos que inician nuevos negocios. Los montos modestos asignados para la asesoría a deudores individuales después de la insolvencia no reconocen la necesidad de una intervención temprana, tal vez en el momento en que se registra la empresa, a fin de brindar capacitación en habilidades básicas sobre gestión financiera y comprensión de los riesgos financieros iniciales de la nueva empresa"; SARRA, cit. (n. 16), p. 3.

18 "Tienen importancia para las mipymes las reglas legales formales y las reglas sociales informales y las normas prácticas que afectan a los empresarios, incluyendo el diseño de las leyes concursales, la estructura de los mercados de capital y la percepción del estigma relativo a la responsabilidad personal”. DAVIS, Ronald B. Madaus, Stephan - Mazzoni, Alberto - Mevorach, Irit - Mokal, Riz - Romanine, Barbara - SARRA, Janis P. - Tirado, Ignacio, The Modular Approach to Micro, Small, and Medium Enterprise Insolvency (2016), pp. 22, disponible [en línea]: https://ssrn. com/abstract $=2904858$.

19 " $L a s$ personas naturales pueden efectuar iniciación de actividades ante el Servicio de Impuestos Internos para los efectos de acogerse a los regímenes de tributación que esa misma disposición establece (artículo 14 del Decreto Ley $\mathrm{N}^{\circ}$ 824, sobre Ley sobre Impuesto a la Renta), sin que, por ello, para otros efectos que no sean los descritos en dicha disposición pueda considerarse que constituyen una persona jurídica distinta a la natural". I. Municipalidad de Concepción con Ida Marsano Crovetto, Corte de Apelaciones, 10 de marzo de 2017 (recurso de apelación), Rol No 293-2016. 
tipo societario que exija capital mínimo. Tampoco la Empresa Individual de Responsabilidad Limitada (EIRL) exige este requisito ${ }^{20}$. Además, las EMT enfrentan obstáculos para acceder al crédito y también tasas de interés muy altas debido al riego de incumplimiento, falta de colateral y/o garantías reales o personales, y falta de historial crediticio ${ }^{21}$. Estas circunstancias obligan a la EMT a recurrir al patrimonio del empresario, principalmente, en su etapa inicial ${ }^{22}$.

Como se ha indicado, la legislación chilena no aborda la formación de la masa activa de la liquidación. En cambio, el soft law presenta una regulación. Por lo tanto, se evaluará el Informe más reciente presentado por la Comisión de las Naciones Unidas para el Derecho Mercantil Internacional (CNUDMI), emanado del Grupo de Trabajo V (Régimen de Insolvencia) sobre la labor realizada en su $55^{\circ}$ período de sesiones en Nueva York el $2019^{23}$. Tal documento analiza, para el caso de las mipymes,

${ }^{20}$ Sobre el punto, vid. Goldenberg, Juan Luis, Mecanismos de protección a los acreedores de una sociedad de responsabilidad limitada infracapitalizada. Análisis critico y propuesta de solución, en Revista Chilena de Derecho Privado 27 (2016), pp. 141225.

${ }^{21}$ Ante esta realidad, la OCDE indica que es posible identificar una serie de políticas emergentes de los gobiernos tendentes a facilitar el acceso a distintas fuentes de financiamiento para la pyme. OCDE. 2018. Financiamiento de pymes $y$ emprendedores, cit. (n.1). Por ejemplo, en Chile, el proyecto de ley (Boletín 10.78503) aprobado por el Congreso Nacional el 21 de diciembre de 2018, que modifica la Ley $\mathrm{N}^{\circ} 19.983$, que regula la transferencia y otorga mérito ejecutivo a la copia de la factura, establece en el artículo $2^{\circ}$ que la obligación de pago del saldo insoluto contenido en la factura deberá ser cumplida de manera efectiva en el plazo máximo de treinta días corridos contado desde la recepción de la factura.

${ }^{22}$ En ocasiones, el apoyo financiero se consigue a través del recurso a otros tipos de personas naturales que actúan como "socios ocultos". Sobre el punto, es posible mencionar un pronunciamiento de la jurisprudencia italiana por el Tribunal de Nápoles Norte, del 11 de mayo 2017 en: Il diritto fallimentare e delle società commerciali. Rivista di dottrina e giurisprudenza 6 (2017), p. 1525. Este tema es ampliamente abordado por la doctrina italiana. Como referencia general, puede consultarse: Galgano, cit. (n. 13), p. 55-59. En Chile, entre otros casos, es posible considerar el reconocimiento de la figura del socio oculto en la sentencia: Constructora Motruns Ltda. con Sandoval, $2^{\circ}$ Juzgado de Letras de Los Andes, 26 de octubre de 2012, (demanda de rendición de cuentas), Rol No C-1498-2010.

${ }^{23}$ En el $52^{\circ}$ período de sesiones, en Viena, 8 a 19 de julio de 2019, la Comisión de las Naciones Unidas para el Derecho Mercantil Internacional (https://uncitral. un.org/es/commission) presentó el Informe del Grupo de Trabajo V (Régimen de Insolvencia) sobre la labor realizada en su $55^{\circ}$ período de sesiones (Nueva York, 28 a 31 de mayo 2019) [visible en internet: https://undocs.org/es/A/CN.9/972] Parte de este Informe contiene un "Proyecto de texto sobre un régimen simplificado de la 
las disposiciones de la Guía Legislativa de la CNUDMI sobre el Régimen de la Insolvencia ${ }^{24}$.

El Informe del Grupo de Trabajo V contiene un acápite dedicado a los "Bienes que constituyen la masa de la insolvencia de las microempresas y pequeñas empresas deudoras" 25 . El texto discurre a partir del razonamiento que la microempresa y la pequeña empresa es una persona natura ${ }^{26}$. Se indica que: "A lo largo del tiempo, la mayoría de los ordenamientos jurídicos ha ido ampliando el perímetro de los bienes excluidos de la masa de la insolvencia, con el fin de brindar a los deudores la oportunidad de comenzar de nuevo" ${ }^{27}$. Este planteamiento implica que, tanto el patrimonio de la EMT como aquel de la persona natural, integran la masa del concurso de la empresa y que las legislaciones deben definir qué bienes quedan excluidos. En este sentido: "La exclusión de dos categorías particulares de bienes, la vivienda familiar y los instrumentos de trabajo, es especialmente importante para reducir el estigma social de la insolvencia y sus efectos en toda la familia y en las posibilidades de restablecimiento de una microempresa o pequeña empresa deudora" ${ }^{28}$.

La consideración de la intersección entre el patrimonio de la mipyme y

insolvencia de la MIPYME”, disponible [en línea]: https://undocs.org/sp/A/CN.9/ WG.V/WP.166.

${ }^{24}$ UnCITRAL, Guia Legislativa de la CNUDMI sobre el Régimen de la Insolvencia, Partes Primera y Segunda (2004), disponible [en línea]: http://www.uncitral.org/ pdf/english/texts/insolven/05-80722_Ebook.pdf. UnCITRAL, Guía Legislativa de la CNUDMI sobre el Régimen de la Insolvencia, Tercera Parte: tratamiento de los grupos en insolvencia (2010), disponible [en línea]: http:/www.uncitral.org/pdf/english/ texts/insolven/Leg-Guide-Insol-Part3-ebook-E.pdf]. UnciTral, Guia Legislativa de la CNUDMI sobre el Régimen de la Insolvencia, Cuarta Parte (2013), disponible [en línea]: http://www.uncitral.org/uncitral/en/uncitral_texts/insolvency/2004Guide. html. El objetivo de la Guía es asistir y ser usada como referencia por las autoridades nacionales y cuerpos legislativos cuando preparan nuevas leyes y regulaciones o revisan la adecuación de aquellas existentes. Las sugerencias contenidas en la Guía pretenden obtener un balance entre la necesidad de acometer las dificultades financieras del deudor, de la manera más rápida y eficiente posible, y el interés de las diversas partes directamente concernidas con tales dificultades financieras, principalmente acreedores y otras partes con un interés en el negocio del deudor, así como también el interés público comprometido.

${ }^{25}$ Acápite V, puntos 123 y ss. Informe del Grupo de Trabajo V (Régimen de Insolvencia), cit. (n. 23), p. 31. Sobre este punto se pronuncia también: INTERNATIONAL Bank for Reconstruction and Development, cit. (n. 15), p. 15.

${ }^{26}$ Esto se advierte claramente en el punto 127 del texto, donde hay una prevención en el sentido que, cuanto se ha indicado, sea aplicado al caso en que la micro y la pequeña empresa sea una persona jurídica.

${ }^{27}$ Punto 123.

${ }^{28}$ Ibid. 
aquel de la persona natural es una perspectiva basada en la realidad, ya que la persona natural suele ofrecer garantías en favor de la empresa, o bien, hay créditos recíprocos. Por lo tanto, según el Informe de la CNUDMI, la evaluación del patrimonio concusal de la mipyme es holística, es decir, el patrimonio personal o las garantías usadas por personas naturales para sostener el negocio deben ser incluidas en el concurso de la empresa ${ }^{29}$. Se considera que tal perspectiva es necesaria, porque el análisis del balance de la empresa -para determinar si es insolvente- podría ser insuficiente si no incluye el pasivo de los propietarios de la mipyme. Sólo una aproximación integral puede revelar el verdadero estado de la empresa. Asimismo, la consideración de bienes del empresario es necesaria, porque su pertenencia, al menos aparente, al patrimonio de la empresa fue un factor que influyó en la evaluación del riesgo del crédito efectuado por los acreedores ${ }^{30}$. En efecto, la Guía Legislativa sobre el Régimen de la Insolvencia indica: "ciertos Estados tienen leyes concursales que procuran distinguir entre aquellos que son simples deudores consumidores y aquellos cuyas obligaciones surgen de pequeños negocios. Puesto que créditos de consumo son comúnmente usados para financiar pequeñas empresas, ya sea como capital start-up o como fondos de operación, no siempre es posible separar las deudas en categorías definidas" ${ }^{31}$. Por lo tanto, este trabajo sostiene la posición en el sentido que el juez del concurso debe considerar los bienes del deudor, en cuanto persona natural, siempre que hayan sido utilizados

${ }^{29}$ Bajo esa perspectiva, de acuerdo al Informe, la exclusión de determinados bienes puede operar, ya sea a través de una solicitud formulada por el deudor hasta un límite de valor determinado, ya sea a través del establecimiento de la exclusión respecto a determinadas categorías de bienes y la fijación de un límite de valor específico para cada una de ellas. Una última posibilidad es la exclusión general de bienes personales sujeta a la impugnación de los acreedores.

${ }^{30} \mathrm{La}$ doctrina italiana se ha pronunciado acerca de la llamada "concesión abusiva del crédito" a una empresa incapaz de pagar regularmente sus deudas. Se considera que, la concesión del crédito a una empresa de estas características acrecienta el riesgo de insolvencia y, por lo tanto, perjudica a los demás acreedores. Así, éstos pueden ejercer pretensiones resarcitorias en contra del Banco. Castronovo no está de acuerdo con esta posibilidad, porque implicaría una prueba por parte del acreedor relativa a la conducta "abusiva" del banco, la cual no es posible, a su juicio. Con todo, la Casación italiana indica que, "el bonus argentarius debe impedir que se utilice una cuenta corriente como instrumento de engaño a los acreedores, y proceder al cierre de la cuenta del cliente de dudosa solvencia", en vez de "aprovecharse de los ingentes intereses debitados al descubierto". La sentencia de Casación corresponde al 13 de junio de 1993, n. 343, en Banca, Borsa e Titoli di Credito, 1994, II, 263 s. Véase: Castronovo, Carlo, La nuova responsabilità civile (Milano, Giuffrè, 2006), pp. 124126.

${ }^{31}$ UnCITRAL, cit. (n. 24), p. 284. 
para el giro empresarial. Con todo, la inclusión de los bienes, créditos y las garantías personales otorgadas por el empresario-persona natural a la empresa es difícil de implementar en el caso de una EMT que ha adoptado una estructura societaria, porque la consideración de bienes o garantías personales en el concurso de la persona jurídica implicaría una confusión de patrimonios que requiere obviar la personalidad jurídica, la cual, en principio, es insoslayable. Sobre el particular se pronuncia el Informe del Grupo de Trabajo V (Régimen de Insolvencia), específicamente, en el punto 127, el cual hace referencia a las Recomendaciones 38 y $109 \mathrm{de}$ la Guia Legislativa ${ }^{32}$, en el siguiente sentido: "se invita a los Estados a considerar la posibilidad de hacer extensivas las medidas contempladas en las recomendaciones 38 y 109 a las microempresas y pequeñas empresas deudoras con personalidad jurídica, reconociendo que independientemente de la forma en que operen y de que gocen o no de la protección que brinda la responsabilidad limitada ${ }^{33}$, en ese tipo de empresas los activos y el patrimonio personal pueden estar entremezclados hasta tal punto que los acreedores podrían seguir reclamando bienes personales de sus propietarios y de otras personas allegadas" ${ }^{34}$. Por lo tanto, es necesario encontrar una

${ }^{32}$ La Guía Legislativa sobre el Régimen de Insolvencia en las Recomendaciones 38 y 109 se refiere de manera muy genérica a los bienes que podrían quedar excluidos de la masa de la insolvencia cuando el deudor es una persona física. La Recomendación 38 dice: "La ley concursal deberá especificar los bienes, si es que existen, que se excluyen del patrimonio cuando el deudor es una persona natural". La Recomendación 109 indica: "Cuando es deudor es una persona natural, la ley concursal debería especificar que el deudor está habilitado para retener aquellos bienes excluidos del patrimonio por ley". UnCiTral, cit. (n. 24), p. 83 y 172.

${ }^{33}$ Las opciones que se brindan a las microempresas y pequeñas empresas para limitar su responsabilidad varían de una jurisdicción a otra. El Grupo de Trabajo I (MIPYME), en particular en el contexto de su labor sobre una entidad de responsabilidad limitada de la CNUDMI (ERL-CNUDMI), actualmente, está examinando mecanismos para la separación de bienes y la limitación de la responsabilidad de las empresas con personalidad jurídica o sin ella; disponible [en línea]: https://undocs.org/es/A/CN.9/968.

${ }^{34}$ Respecto a la relación entre el Informe de CNUDMI y la Guía Legislativa, es posible indicar que, en su $49^{\circ}$ período de sesiones, en 2016, la Comisión aclaró el mandato que había otorgado al Grupo de Trabajo V (en 2013) con respecto a la insolvencia de las MIPYME de la siguiente manera: "Se encomienda al Grupo de Trabajo V el mandato de desarrollar soluciones y mecanismos adecuados, centrándose tanto en las personas naturales como jurídicas que se dedican a actividades comerciales, para resolver la insolvencia de las MIPYME. Si bien los principios fundamentales de la insolvencia y la orientación proporcionada por la Guía legislativa de la CNUDMI sobre el régimen de la insolvencia deberían ser el punto de partida de los debates, el Grupo de Trabajo debería tratar de adaptar los mecanismos ya previstos en la Guía legislativa para abordar específicamente las 
vía para aplicar las recomendaciones del Informe del Grupo de Trabajo $\mathrm{V}$, en cuanto a la relación patrimonial de la empresa y el empresario, cuando éste ha decidido otorgar forma societaria a su empresa, o bien, ha constituido una EIRL ${ }^{35}$.

En línea con lo planteado, el Report on the Treatment of MSME Insolvency reconoce la confusión entre el patrimonio de la empresa y aquel del empresario, aun cuando la empresa haya asumido una forma societaria: "Donde los regímenes lo permiten, los acreedores han desarrollado una solución para gestionar el riesgo comercial de negociar con el tipo de mipyme donde existe un alto nivel de envolvimiento con los negocios personales del empresario y/o bienes personales de mayor valor que aquellos de la empresa. En esas circunstancias, los acreedores buscan traer los bienes personales del empresario al radio de alcance del concurso de la mipyme a través de garantías personales y derechos reales sobre bienes personales. Una garantía personal normalmente extiende la responsabilidad por las deudas de la mipyme al empresario; el efecto de esto es que los bienes personales como también aquellos de la empresa de propiedad del empresario pueden ser afectados por la insolvencia de la mipyme (por lo tanto, incluso si la mipyme es una persona jurídica, el velo corporativo, efectivamente, se levanta)" ${ }^{36}$.

Este artículo, acepta el planteamiento del Report y, además, sostiene que, aun cuando los acreedores no hayan constituido garantías sobre bienes personales del empresario, igualmente podrían hacer efectivos sus créditos sobre dichos bienes, si forman parte del giro de la empresa, a través del levantamiento del velo societario ${ }^{37}$.

cuestiones relacionadas con las MIPYME...", disponible [en línea]: https://undocs. org/sp/A/71/17.

${ }^{35}$ En América Latina muchas pymes tienden a realizar sus actividades en la economía informal. Vid. Organización Internacional del Trabajo, Formalización de las pymes en las cadenas de valor en América Latina: ¿cuál es la función de las empresas multinacionales? (2016), disponible [en línea]: https://www.ilo.org/wcmsp5/groups/ public/---ed_emp/---emp_ent/---ifp_seed/documents/publication/wcms_548320. pdf. Desde el punto de vista económico y social, la formalización de la pyme es conveniente para fortalecer su productividad en los mercados nacionales y ampliar su acceso a los mercados internacionales. Asimismo, le permite su interacción con otras pymes, con las grandes empresas y con instituciones financieras tanto públicas como privadas. Vid. Valenzuela, cit. (n. 4).

${ }^{36}$ International Bank for Reconstruction and Development, Report on the Treatment, cit. (n. 15), pp. 16.

${ }^{37}$ Levantar el velo significa abolir el sistema de responsabilidad limitada que el individuo creó a través de la adopción de la fórmula societaria o como EIRL. Se extiende la responsabilidad patrimonial de la empresa hasta sus confines reales, más allá de la formalidad. 
Para obtener el levantamiento del velo se requiere probar que, en los hechos, existe una empresa, la cual abarca parte del patrimonio del empresario $^{38}$. Esta solución se considera adecuada, porque lo que ocurre en la realidad es que el empresario usa dinero o bienes personales para financiar el negocio sin que necesariamente se documenten esos traspasos patrimoniales como un mutuo a la empresa o de otra forma. Asimismo, es muy frecuente que el dinero que el empresario invirtió en la empresa provenga de un mutuo que éste obtuvo de un acreedor, quien espera que la persona natural le pague, no la pyme, aun sin constituir garantías.

En materia de levantamiento del velo corporativo, los tribunales de justicia chilenos han dictaminado "[q]ue el abuso de la forma societaria y la consiguiente aplicación de la técnica judicial del levantamiento del velo tienen lugar ante la presencia de dos supuestos copulativos: primero, la identidad personal o patrimonial entre una sociedad y uno o más de sus socios, administradores o sociedades relacionadas; y, segundo, la instrumentalización abusiva de tal sociedad para la consecución de un fraude a la ley o a los derechos de terceros" 39 . Por lo tanto, se invoca la idea del abuso como principio de base que justifica el levantamiento del velo $^{40}$. En el caso del traspaso de bienes, garantías y créditos que tuvo lugar entre el patrimonio del empresario y aquel de la mipyme no existe ánimo de defraudar. Antes bien, el empresario apoyó a la empresa para

${ }^{38}$ Como es sabido, el levantamiento del velo ha experimentado una evolución. En principio, se utilizó para llegar al patrimonio personal del socio y hoy se utiliza en el caso de grupos de empresas para llegar a la matriz, probando que, en los hechos, las sociedades del grupo conforman una empresa. Vid. JeQuier, Eduardo, Premisas para el tratamiento de los grupos empresariales y administradores de hecho en el Derecho chileno, en Revista Chilena de Derecho 41 (2014), 1, pp. 121-152.

39 "Muñoz con Compañía de Telecomunicaciones de Chile S.A.", 7o Juzgado Civil de Santiago, 24 de octubre de 2013 (indemnización de perjuicios por responsabilidad contractual), Rol No C-17535-2009. En esa línea, se pronuncia UGARTE VIaL, Jorge, Fundamentos y acciones para la aplicación del levantamiento del velo en Chile, en Revista Chilena de Derecho 39 (2012), 3, pp. 699: "se define el levantamiento del velo como un procedimiento judicial de carácter excepcional, en virtud del cual cabe prescindir en ciertos casos de la separación personal y patrimonial entre una sociedad de cuya estructura jurídica se ha abusado, por una parte, y uno o más de sus socios, administradores o sociedades relacionadas, por otra, para evitar así que un fraude o abuso produzca efectos".

${ }^{40}$ Los tribunales en los Estados Unidos también invocan el abuso como fundamento del levantamiento del velo. Vid. Cheng Han, Tan - Jiangyu, Wang - Hofmann, Christian, Piercing the Corporate Veil: Historical, Theoretical and Comparative Perspectives, en NUS Law Working Paper 2018/025. NUS - EW Barker Centre for Law \& Business Working Paper 18/04 (2018), p. 13. 
que ésta lograra desarrollarse ${ }^{41}$. Por lo tanto, en principio, la integración patrimonial es difícil de alcanzar mediante la fórmula del levantamiento del velo. Sin embargo, en este caso, mantener la separación estricta entre el patrimonio social y aquel del empresario generaría injusticia en el contexto de la insolvencia ${ }^{42}$. La personalidad separada no puede extenderse hasta un punto más allá de su ratio ${ }^{43}$. Así, la teoría de la personalidad jurídica se ha desarrollado en el sentido que su utilidad radica en la materialización de políticas esenciales del Estado, como la promoción del comercio y el crecimiento industrial ${ }^{44}$. En tal condición, "[n]o se puede afirmar para un propósito que no promueva estos objetivos, con el fin de sobreponerse a otros intereses públicos significativos que el Estado busca proteger a través de la legislación o regulación" ${ }^{5}$. Por lo tanto, se ha afirmado la idea que la sociedad con personalidad jurídica es un conjunto de individuos que actúan bajo una ficción jurídica que la ley ha introducido por la conveniencia de conducir los negocios de esta forma privilegiada. En dicha concepción está implícito que hay límites para esta separación entre la persona jurídica y los socios o empresarios. Los límites están determinados por el objeto del sistema jurídico de establecer fines sociales y económicos positivos, a través

${ }^{41}$ Generalmente, el empresario se ve impelido a sustentar el andamiento de la empresa con su patrimonio, porque constituyó una sociedad con capital insuficiente. Desde ese punto de vista, el levantamiento del velo aparece, en principio, justificado. En los hechos, hasta 2007, la doctrina en Alemania discutía si la infracapitalización de la $G m b H$ justificaba el levantamiento del velo. Ese año la Corte Suprema falló contra la aplicación de esta solución. CHENG HAN, cit. (n. 40), p. 32.

42 "En Vuitch v Furr la Corte sostuvo que la insolvencia o infracapitalización es a menudo un factor importante que evidencia injusticia” CHENG Han, cit. (n. 40), p. 27, n. 153.

${ }^{43}$ En esa línea: "Consorcio Allianz de Seguros Generales con Sociedad Naviera Ultrajas Ltda. y Ultramar Agencia Maritima Ltda. ": Corte Suprema, de 2 de junio 2009 (recurso de casación en el fondo), Rol N ${ }^{\circ}$ 1527-2008. En este caso, los tribunales se vieron en la necesidad de aplicar el levantamiento del velo, aun sin ser solicitado por los demandantes, "puesto que, de lo contrario, a juicio de la Corte Suprema, se habrian vulnerado otros principios generales de nuestra legislación, tales como la supremacía de la realidad y la buena fe". Vid. el análisis de la sentencia en: Urbina Molfino, Ignacio, Levantamiento del velo corporativo, en Revista Chilena de Derecho 38 (2011), 1, pp. 163-171. La cita corresponde a la p. 164.

${ }^{44} \mathrm{La}$ persona jurídica tiene origen en el trabajo de los canonistas. Esta creación se desarrolló por la Pandectística alemana del siglo XVIII y XIX. Vid. AraYA, Cristian, La Máscara Social, en Revista de Derecho de la Universidad Católica de Valparaíso 16 (1995), p. 47.

45 "Glazer v. Commission on Ethics for Public Employees", 431 So. 2d 752. Supreme Court of Louisiana, 4 april 1983, disponible [en línea]: https://law.justia.com/cases/ louisiana/supreme-court/1983/82-c-1853-1.html. 
de la organización de un marco que facilite las transacciones comerciales. En el caso de la constitución de la masa activa de la liquidación, la mantención de la personalidad jurídica, aun cuando existe una empresa que excede dicho ropaje jurídico, produciría un entorpecimiento al comercio. Por lo tanto, la fórmula que se propone es levantar el velo para obtener el reconocimiento del sustrato empresarial que, en los hechos, opera. Tal operación permite dar cabida en el concurso a los créditos o garantías otorgados por el empresario con su patrimonio personal y, también, incluir los bienes que éste aportó informalmente para el desarrollo del giro ${ }^{46}$.

Lógicamente, los afectados ante el levantamiento del velo de la EMT son los acreedores personales del deudor, quienes pueden surgir con posterioridad a la constitución de la empresa individual, o bien, pueden existir al momento de la constitución de la empresa. En el primer caso, el análisis del riesgo del crédito debería comprender el estudio del nivel de recursos aportados al patrimonio de la EMT, para evitar que la infracapitalización devenga en el levantamiento del velo ${ }^{47}$. En el segundo caso, es decir, cuando la empresa se constituye con posterioridad a la asunción de la deuda personal, los acreedores no tienen forma de prever esta situación y, consecuentemente, no pueden protegerse ante la "amenaza" que ello implica para su crédito. Con todo, este estudio mantiene, conforme a la explicación que sigue, que, la integración al patrimonio concursal de aquellos bienes pertenecientes al patrimonio personal -los cuales, en los hechos, forman parte de la empresa-, no significa un atentado al derecho de garantía general de los acreedores personales.

La noción tradicional del patrimonio indica que los activos están sujetos a la acción ejecutiva de los acreedores ${ }^{48}$. Sin embargo, en la actualidad, todo apunta a la especialización de la responsabilidad patrimonial. Los sistemas jurídicos modernos contemplan la posibilidad de fragmentar el patrimonio personal, a través de la constitución de Empresas Individuales de Responsabilidad Limitada, o bien, a través de modelos societarios de un solo socio. Aun las sociedades pueden fragmentar su patrimonio a través de la creación de patrimonios destinados ${ }^{49}$. Estos fenómenos constituyen

\footnotetext{
${ }^{46}$ En esta línea, UnCITRAL, cit. (n. 24), p. 95, punto 12.

${ }^{47}$ Vid. Gelb, Harvey, Piercing the Corporate Veil. The Undercapitalization Factor, en Chicago Kent Law Review 59 (1982), pp. 1-22, especialmente, p. 3

${ }^{48}$ Este es el llamado derecho de garantía general de los acreedores, el cual no significa prohibir al deudor la ejecución de actos de disposición. Bianca, Cesare Massimo, Diritto Civile. La responsabilità (Milano, Giuffrè, 1994), V, p. 435, opina que la limitación al poder de disposición del deudor sería una consecuencia excesiva respecto a la exigencia de tutela de los acreedores.

49 "De patrimonio separado, autónomo, dedicado o segregado se habla en
} 
una desviación del principio general de la responsabilidad patrimonial o derecho de garantía general de los acreedores. Un autor incluso opina que, más que una desviación, son la vía hacia el ocaso de la responsabilidad ${ }^{50}$. Otro autor indica que, "en la doctrina del patrimonio separado puede advertirse una evolución del proceso económico que sustituye al hombre, como protagonista de la escena social, por el capital y que promueve una concepción orgánica de la empresa... Así, el jurista intenta conciliar los institutos del Derecho privado, elaborados por las categorías romanísticas, con aquellos del emergente Derecho de la empresa" ${ }^{51}$. Por lo tanto, el Derecho ha asumido -y los acreedores acatado- una dirección relativa a la formación de patrimonios separados o de destinación de activos para el desarrollo de una actividad empresarial. Ante esta situación, los acreedores personales sólo pueden precaverse a través de la constitución de garantías,

todas las ocasiones en que la ley considera un determinado núcleo patrimonial como objeto de una disciplina jurídica particular y se registra una desviación del principio general de la responsabilidad patrimonial"; Zoppini, Andrea, Autonomia e separazione del patrimonio, nella prospettiva dei patrimoni separati della società per azioni, en Rivista di Diritto Civile (2002), 1, pp. 545-575. La cita corresponde a la p. 552. En Chile, el artículo 138 de la Ley 18.045 de Mercado de Valores indica: "Los acreedores generales de la sociedad, cualquiera sea el origen o calidad de sus créditos, no podrán hacerlos efectivos en los bienes que conformen el activo del o de los patrimonios separados constituidos por su deudor ni afectarlos con gravámenes, prohibiciones, medidas precautorias o embargos, sino sólo cuando hayan pasado a integrar el patrimonio común en los casos que se permiten en este título. Sobre los activos que integren un patrimonio separado, sólo puede perseguirse el pago de las obligaciones que provengan de los títulos de deudas emitidos con cargo al mismo, sin perjuicio de lo establecido en el inciso cuarto de este articulo".

${ }^{50}$ LoPucki, Lynn M., The death of liability, en Yale Law Journal 106 (19961997), pp. 1-91. Según el autor (en p. 7), las causas de la decadencia del sistema de responsabilidad son las siguientes: “(1) el sistema no está dispuesto a bloquear la participación de aquellos sin recursos suficientes en actividades generadoras de responsabilidad; y (2) al sistema le falta un marco conceptual efectivo para atribuir riqueza a aquellos comprometidos en actividades que producen responsabilidad. Estableciendo un nivel modesto de responsabilidad financiera para todos aquellos comprometidos en actividades que generan responsabilidad podría conservar el sistema de responsabilidad, pero sólo como una aparición de los que era su esencia anterior" (p. 7). "Pienso en el sistema de la responsabilidad como un juego de póker... Pronto nadie tendrá suficientes fichas en el recipiente. Cuando esto ocurra la naturaleza esencial del juego cambiará. La responsabilidad morirá” (p. 3). Es necesario indicar que el autor considera "en riesgo" sólo la responsabilidad extracontractual y aquella legal. La responsabilidad contractual está asegurada, precisamente, por medio de las cláusulas pactadas entre las partes.

${ }^{51}$ Zoppini, cit. (n. 49), p. 557. 
o bien, por medio de la concesión de créditos con plazos acotados respecto de aquellos acreedores con solvencia limitada ${ }^{52}$.

En suma, el velo se levanta para formar la masa activa de la liquidación, porque, en los hechos, opera una empresa que se sirve de los bienes empresariales y de aquellos personales del empresario. Para que el juez pueda efectuar este ejercicio requiere identificar a la empresa. Por lo tanto, se necesita contar, ante todo, con una noción de empresa que sea omnicomprensiva del fenómeno. Esa noción se examina en el apartado que sigue.

\section{El concepto de Empresa de Menor Tamaño insolvente}

La calificación de micro, pequeña o mediana empresa que la Ley $\mathrm{N}^{\circ}$ 20.416 efectúa en el Artículo Segundo se conforma con un criterio cuantitativo, extrapolado del Derecho tributario, y basado en ingresos anuales por ventas y servicios y otras actividades del giro. Tal aproximación a la EMT es válida ${ }^{53}$. pero no revela en plenitud el fenómeno de la Empresa de Menor Tamaño insolvente. Una descripción acabada del fenómeno permitiría solucionar dificultades y llenar vacíos que la práctica mercantil presenta. Un autor, a propósito de la Ley de Insolvencia y Quiebra de Canadá, plantea una alternativa a la consideración de los ingresos anuales, conforme al volumen de ventas y servicios y otras actividades del giro ${ }^{54}$ : “[e]l acceso al proceso de reorganización de la mipyme puede estar determinado por una combinación de número de empleados y un límite de deudas"55. Naturalmente, estos criterios $-\mathrm{u}$ otros que pudieren utilizarse para identificar la empresa en insolvencia- difieren dependiendo del contexto y del

${ }^{52} \mathrm{Vid}$. Goldenberg, Juan Luis, El necesario ajuste de la asignación del riesgo de sobreendeudamiento en la regulación de las tarjetas de crédito: desde un sistema basado en los deberes de información a un modelo de corresponsabilidad, en Revista de Derecho de la Pontificia Universidad Católica de Valparaíso 49 (2017), 2, pp. 55-98. Vid. también: Logemann, Jan, From cradle to bankruptcy? Credit Access and the American Welfare State, ahora, en LogEMANn, Jan (editor), The development of consumer credit in global perspective. Business, regulation, and culture (New York, Palgrave Macmillan, 2012), pp. 201-219.

${ }^{53} \mathrm{La}$ Unión Europea utiliza indicadores similares. Conforme a la Comisión Europea, el volumen de ventas de una microempresa ha de ser $\leq € 2 \mathrm{~m}$; de una pequeña empresa $\leq € 10 \mathrm{~m}$; y de una mediana empresa $\leq € 50 \mathrm{~m}$. La Comisión Europea considera, además, el número de trabajadores. European Commission, What is an SME? Entrepreneurship and SMEs (2018), disponible [en línea]: http:// ec.europa.eu/growth/smes/business-friendly-environment/sme-definition_en.

${ }^{54}$ Bankruptcy and Insolvency Act (BIA) 1985.

${ }^{55}$ SARra, cit. (n. 16), p. 2. 
lugar en que se usa ${ }^{56}$. El Informe del Grupo de Trabajo V da cuenta que, en el seno de tal Grupo de Trabajo, "se expresaron distintas opiniones sobre la factibilidad y la conveniencia de que se elaborara una definición de microempresas y pequeñas empresas. La opinión prevaleciente fue que cada jurisdicción debía tratar esa cuestión en su derecho interno" ${ }^{77}$. Tales empresas pueden encontrarse en un espectro amplio y heterogéneo. Por lo tanto, es natural que tal instrumento no ofrezca una definición que las abrace a todas.

Para delimitar un concepto de empresa funcional a los fines establecidos en este artículo, es decir, que permita comprender su patrimonio efectivo, es necesario remitirse, ante todo, a una definición general de empresa. Esta es una noción referida a la actividad económica organizada de producción y circulación de bienes y servicios para el mercado, ejercida profesionalmente. Además, se considera que la empresa es una unidad de producción e intercambio cuya eficiencia y contribución al bien común es deseable ${ }^{58}$. Si bien todos los elementos descritos son válidos para identificar a la mipyme, ésta presenta una particularidad sine qua non: está centrada en el trabajo humano, antes que en el capital. De hecho, el Capítulo 2 del Report on the Treatment of MSME Insolvency, indica que existen diferentes definiciones de mipymes dependiendo del contexto y del lugar en que se usa. El mismo estudio indica que el criterio relativo al número de trabajadores es el método común para distinguir las mipymes de las grandes empresas ${ }^{59}$. Otro estudio del Banco Mundial indica que, "[l]as definiciones más comunes utilizadas por los reguladores se basan en el

${ }^{56}$ Por ejemplo, un aspecto común de las EMT, que pone de relieve la Corte Suprema de Chile, es que estas "entidades carecen, por sí mismas, del poder negociador". "Procedimiento para la dictación de instrucción general sobre los efectos en la libre competencia de la diferenciación de precios en los servicios públicos de telefonía 'tarifas on net / off net' $y$ de las ofertas conjuntas de servicios", Corte Suprema, 17 diciembre 2013 (recurso de reclamación), Rol N² 2506-2013.

${ }^{57}$ Informe del Grupo de Trabajo V (Régimen de la Insolvencia) sobre la labor realizada en su $55^{\circ}$ período de sesiones (Nueva York, 28 a 31 de mayo de 2019), cit. (n. 23), p. 10, Punto $\mathrm{N}^{\circ} 29$.

${ }^{58}$ Así, Carvajal, cit. (n. 14), p. 95. Sobre la contribución de la empresa al bien común, vid. BOUCKAERT, Luk, Spiritual humanism and corporate economics, en BRUNI, Luigino - Zamagni, Stefano (eds.), Handbook on the Economics of Reciprocity and Social Enterprise (Cheltenham, 2013), pp. 344-353.

${ }^{59}$ En Chile se utiliza un criterio de este tipo en el Código del Trabajo en el artículo 505 bis (modificado por la Ley N 20.416 de 2010). Para los efectos de este Código y sus leyes complementarias, los empleadores se clasificarán en micro, pequeña, mediana y gran empresa, en función del número de trabajadores. Se entenderá por microempresa aquella que tuviere contratados de 1 a 9 trabajadores, pequeña empresa aquella que tuviere contratados de 10 a 49 trabajadores, mediana 
número de trabajadores, ventas y/o el volumen de deudas por mutuos. El criterio más común entre los tres indicados es el número de trabajadores" ${ }^{\prime \prime}$. En esta misma línea, la Corte Suprema de Chile en una sentencia de 2013 indica que los criterios que identifican a la pyme son su patrimonio y el número de trabajadores ${ }^{61}$. Además, el liderazgo del propietario y/o de un pequeño grupo de copropietarios es irremplazable. Por lo tanto, es claro que el valor inherente en un negocio de este tipo es la productividad humana, la cual no es transferible, como los bienes tangibles o intangibles que forman parte de una empresa grande que deviene insolvente.

El Proyecto de Ley que establece medidas para impulsar la productividad y el emprendimiento, el cual pretende modificar la Ley $\mathrm{N}^{\circ} 20.720$ en lo tocante a las mipymes, contempla incorporar a dicha Ley el concepto de Empresa de Menor Tamaño ${ }^{62}$. Así, el Proyecto entiende por tal, toda empresa que corresponda a la categoría de Micro, Pequeña y Mediana Empresa en conformidad a lo dispuesto por el Artículo Segundo de la Ley $\mathrm{N}^{\circ}$ 20.416, que fija normas especiales para las empresas de menor tamaño ${ }^{63}$. Es decir, se usará como criterio, únicamente, el volumen de ingresos por ventas y servicios y actividades del giro, sin considerar otros elementos, como aquel relativo a la productividad humana -que se ha mencionado.

empresa aquella que tuviere contratados de 50 a 199 trabajadores y gran empresa aquella que tuviere contratados 200 trabajadores o más.

${ }^{60}$ Ardic, Oya Pinar - Mylenko, Nataliya - Saltane, Valentina, Small and Medium Enterprises A Cross-Country Analysis with a New Data Set. The World Bank Financial and Private Sector Development Consultative Group to Assist the Poor, en Policy Research Working Paper 5538 (2011), p. 8.

${ }^{61}$ Ventas anuales: promedio anual cercano a las 25.000 UF y que su número de trabajadores sea inferior a 200. "Ingeniería Montaje y Mantenimiento Eléctricos Limitada con Superintendencia de Electricidad y Combustibles", Corte Suprema, 8 de abril 2013 (recurso de apelación), Rol No 9175-2012.

${ }^{62}$ Boletín $\mathrm{N}^{\circ}$ 12.025-03 Artículo Noveno. Este Artículo ordena, además, eliminar a los profesionales del concepto de empresa deudora contenido en el artículo $2^{\circ} \mathrm{N}^{\circ} 13$ de la Ley $\mathrm{N}^{\circ} 20.720$ : “a) Elimínase en el numeral 13), la frase 'o del número 2) del artículo 42”. Como referencia, el artículo $42 \mathrm{~N}^{\circ} 2$ de la Ley sobre Impuesto a la Renta, establece un impuesto a los ingresos provenientes del ejercicio de las profesiones liberales o de cualquiera otra profesión u ocupación lucrativa no comprendida en la Primera Categoría, ni en el número anterior (rentas del trabajo dependiente), obtenidos entre otros, por sociedades de profesionales que presten exclusivamente servicios o asesorías profesionales.

${ }^{63}$ En el ámbito de la insolvencia de la empresa, el proyecto prevé otras medidas. A saber, se establecen límites a los honorarios del veedor en el caso que se trate de EMT; y, también, se establece que, si la Empresa Deudora califica como una empresa de menor tamaño, podrá acompañar la información relativa a sus acreedores por medio de una declaración jurada. 
El Proyecto de Ley que se tramita presenta la oportunidad de incorporar una noción de Empresa de Menor Tamaño que considere los elementos esenciales de toda empresa. De esta forma, la Ley N ${ }^{\circ} 20.720$ devendría la primera legislación con una definición sustancial de empresa. Además, por cierto, deberían incorporarse los elementos particulares de la mipyme, como el número de trabajadores y la participación del propietario -o de los propietarios- en la administración.

Una vez que el juez identifica los elementos esenciales de la empresa, puede establecer su existencia y, luego, determinar cuál es el patrimonio real de la Empresa de Menor Tamaño en insolvencia. Tal ejercicio implicará que, posiblemente, ciertos activos del empresario-persona natural que, en los hechos, han formado parte del giro de la empresa, pasen a integrar la masa concursal.

\section{CONCLUSIÓN}

En general, en el mundo hay pocos regímenes especializados en la insolvencia de la Empresa de Menor Tamaño. Este fenómeno se trata como la insolvencia de las otras empresas, no obstante las características particulares de la EMT. Este trabajo consideró, entre los numerosos desafíos que enfrenta la legislación nacional en relación a este argumento, aquel relativo a la conformación del patrimonio de la EMT insolvente.

Como es sabido, la gran dificultad de este tema es la confusión entre el patrimonio de la EMT y aquel del empresario o propietarios de la empresa. Los documentos del soft law invitan a generar una mirada holística del patrimonio, cuya consecución en la práctica, conforme a las legislaciones nacionales, es complejo, especialmente en el caso en que la EMT se ha constituido como una sociedad o una EIRL, es decir, con personalidad jurídica. Es esos casos, para aplicar la recomendación del soft law, debe levantarse el velo societario, pues se considera que, más allá del revestimiento de la personalidad jurídica, hay una empresa. Se entenderá por tal la unidad productiva de bienes y servicios para el mercado, cuyo aporte al bien común es deseable y en la cual el factor humano es un requisito sine qua non. La coyuntura -a través de un Proyecto de Ley en tramitación en el Congreso Nacional que pretende introducir una definición de EMT a la Ley $\mathrm{N}^{\circ} 20.720$ - ofrece la oportunidad de contar en la legislación comercial chilena con una definición sustancial de empresa basada en parámetros que presenta el fenómeno de la empresa comercial en la realidad. Esta definición facilitará, ante todo, su identificación por el juez; y, luego, la determinación del patrimonio concursal de la EMT. 


\section{BiBLIOGRAFíA}

Araya, Cristian, La Máscara Social, en Revista de Derecho de la Universidad Católica de Valparaíso 16 (1995).

Ardic, Oya Pinar - Mylenko, Nataliya - Saltane, Valentina, Small and Medium Enterprises A Cross-Country Analysis with a New Data Set. The World Bank Financial and Private Sector Development Consultative Group to Assist the Poor, en Policy Research Working Paper 5538 (2011).

Bianca, Cesare Massimo, Diritto Civile. La responsabilità (Milano, Giuffrè, 1994), V.

BoucKaert, Luk, Spiritual humanism and corporate economics, ahora, en BRUNI, Luigino - Zamagni, Stefano (editores), Handbook on the Economics of Reciprocity and Social Enterprise (Cheltenham, 2013).

Carvajal, Lorena, La codificación del Derecho comercial chileno y sus ejes, en Revista Chilena de Derecho 44 (2017), 1.

Castronovo Carlo, La nuova responsabilità civile (Giuffrè, Milano, 2006)

Cheng Han, Tan - Jiangyu, Wang - Hofmann, Christian, Piercing the Corporate Veil: Historical, Theoretical and Comparative Perspectives, en NUS Law Working Paper 2018/025. NUS - EW Barker Centre for Law \& Business Working Paper 18/04 (2018).

COMisión de eStUdio nUEVA CODIFICACIÓn COMERCial, Sujetos del comercio y de los negocios, la empresa y sus elementos, en particular la propiedad intelectual, tercera subcomisión (Universidad de Chile, Facultad de Derecho, Departamento de Derecho Concursal, 2017).

Comisión de las Naciones Unidas para el Derecho Mercantil Internacional, Informe del Grupo de Trabajo V (Régimen de Insolvencia) (2019).

Davis, Ronald B. - Madaus, Stephan - Mazzoni, Alberto - Mevorach, Irit - MoKal, Riz - Romanine, Barbara - Sarra, Janis P. - Tirado, Ignacio, The Modular Approach to Micro, Small, and Medium Enterprise Insolvency (2016),

European Commission, What is an SME? Entrepreneurship and SMEs (2018).

Galgano, Francesco, Diritto Commerciale. L'emprenditore - Le società (8a edición, Bologna, Zanichelli, 2013).

Gelb, Harvey, Piercing the Corporate Veil. The Undercapitalization Factor, en Chicago Kent Law Review 59 (1982).

Goldenberg, Juan Luis, El necesario ajuste de la asignación del riesgo de sobreendeudamiento en la regulación de las tarjetas de crédito: desde un sistema basado en los deberes de información a un modelo de corresponsabilidad, en Revista de Derecho de la Pontificia Universidad Católica de Valparaíso 49 (2017).

Goldenberg, Juan Luis, Mecanismos de protección a los acreedores de una sociedad de responsabilidad limitada infracapitalizada. Análisis crítico y propuesta de solución, en Revista Chilena de Derecho Privado 27 (2016).

Guerrero, Maribel - Serey, Tomás, Global Entrepreneurship Monitor. Reporte Nacional de Chile (2018).

International Bank for Reconstruction and Development/The World Bank, Report on the Treatment of MSME Insolvency (2017).

JEQUIER, Eduardo, Premisas para el tratamiento de los grupos empresariales y administradores de hecho en el Derecho chileno, en Revista Chilena de Derecho 41 (2014), 1. 
Logemann, Jan, From cradle to bankruptcy? Credit Access and the American Welfare State, en Logemann, Jan (ed.), The development of consumer credit in global perspective. Business, regulation, and culture (New York, Palgrave Macmillan, 2012).

LoPucki, Lynn M., The death of liability, en Yale Law Journal 106 (1996-1997).

Messineo, Francesco, Accordo simulatorio e dissimulazione di contrato, en Rivista di Diritto Civile 12 (1966), 1.

OCDE, Financiamiento de pymes y emprendedores 2018: un marcador de la OCDE (2018).

Organización Internacional del Trabajo, Formalización de las pymes en las cadenas de valor en América Latina: ¿cuál es la función de las empresas multinacionales? (2016).

PugA, Juan Esteban, Derecho concursal. El acuerdo de reorganización (4ª edición, Santiago, Editorial Jurídica de Chile, 2015).

Ruz, Gonzalo, Nuevo Derecho Concursal Chileno (Santiago, Thomson Reuters, 2017).

SARra, Janis, Micro, Small and Medium Enterprise (MSME) Insolvency in Canada. Report for the Marketplace Policy Branch of Industry Canada, en Peter A. Allard School of Law Allard Research Commons, Faculty Publications (2016).

UGaRTE VIaL, Jorge, Fundamentos y acciones para la aplicación del levantamiento del velo en Chile, en Revista Chilena de Derecho 39 (2012), 3.

Uncitral, Guia Legislativa de la CNUDMI sobre el Régimen de la Insolvencia, Partes Primera y Segunda (2004).

UnCITRAL, Guía Legislativa de la CNUDMI sobre el Régimen de la Insolvencia, Tercera Parte: tratamiento de los grupos en insolvencia (2010).

Uncitral, Guía Legislativa de la CNUDMI sobre el Régimen de la Insolvencia, Cuarta Parte (2013).

Urbina Molfino, Ignacio, Levantamiento del velo corporativo, en Revista Chilena de Derecho 38 (2011), 1.

Valenzuela María Elena - Di Meglio, Roberto - ReinecKe, Gerhard, La microempresa en Chile: la importancia del entorno regulatorio y desarrollo social, en VALENZUELA, María Elena - Di Meglio, Roberto - ReineCKe, Gerhard (eds.), De la Casa a la formalidad. Experiencias de la Ley de Microempresas Familiares en Chile (Santiago, Oficina Internacional del Trabajo, 2006).

ZoppINI, Andrea, Autonomia e separazione del patrimonio, nella prospettiva dei patrimoni separati della società per azioni, en Rivista di Diritto Civile (2002), 1. 